\title{
EDITORIAL
}

\section{Contribution of Microbiologists in Nepal during COVID-19 Pandemic}

Coronavirus disease (COVID-19) caused by novel coronavirus initiated from Wuhan China affected the global population and spread in 218 countries and territories. In Nepal, the first case of COVID-19 was reported in 23 January, 2020. Central Department of Microbiology, Tribhuvan University and microbiologists in the country contributed to strengthen laboratory diagnosis of COVID-19 in Nepal from the very beginning of the pandemic in the country. The department participated in COVID-19 control effort of the Nepal Government. Team of four microbiologists collected blood samples from the people in quarantine from Kailali and Kanchanpur districts to validate RDT kit for detection of SARS-CoV-2 infection. The team from the Central Department of Microbiology, Tribhuvan University established SARS-CoV-2 diagnostic laboratory through real time PCR in Karnali Province, Surkhet. Similarly, microbiologists contributed to establish real time PCR laboratories for
SARS-CoV-2 diagnosis in province 5, Bhairahawa, TU Teaching Hospital, province 2 Janakpur and Mechi Kankai laboratory Surunga. Further, the microbiologists also trained laboratory personnels for detecting SARS$\mathrm{CoV}-2$ genes using real time PCR.

Microbiologists involved in creating awareness through series of media interviews to people on coronavirus infection and prevention methods. Microbiologists assessed the COVID-19 diagnostic laboratories of the country and submitted report with recommendations to improve the diagnostic capacities. Microbiologists are working in many diagnostic laboratories of the country to diagnose SARS-CoV-2. Realizing the contribution of microbiologists in COVID-19 pandemic, these molecular diagnostic laboratories should be converted to infectious disease diagnostic and research laboratories in post COVID times and knowledge and skills of the microbiologists should be utilized in the country.

\section{Dr. Megha Raj Banjara}

Chief Editor

Tribhuvan University Journal of Microbiology (TUJM) 